\title{
Diamond particle detector systems
}

\author{
Alexander $\mathrm{Oh}^{* \dagger}$ \\ University of Manchester \\ E-mail: alexander.oh@manchester.ac.uk
}

Diamond detectors have been used in HEP experiments at the LHC and upgrades are foreseen during the shutdown phase before LHC restarts its operation. CVD diamond has been used extensively in beam condition monitors as the innermost detectors in the highest radiation areas of $\mathrm{BaBar}$, Belle, CDF and all LHC experiments, and is also expected to be used in the experiments at FAIR at the GSI. The lessons learned in constructing the ATLAS Beam Conditions Monitor (BCM), Diamond Beam Monitor (DBM) and the CMS Pixel Luminosity Telescope (PLT) all of which are based on CVD diamond with the goal of elucidating the issues that should be addressed for future diamond based detector systems. The first beam test results of prototype diamond devices with $3 \mathrm{D}$ detector geometry should further enhance the radiation tolerance of this material.

The 23rd International Workshop on Vertex Detectors,

15-19 September 2014

Macha Lake, The Czech Republic

\footnotetext{
* Speaker.

${ }^{\dagger}$ Material from the RD42 and ADAMAS collaborations.
} 


\section{Introduction}

The experimental program of the Large Hadron Collider (LHC) at CERN foresees an upgrade of the machine to increase by up to two orders of magnitude the integrated luminosity relative to the luminosity collected during run-1. The increased luminosity poses challenges to the detector technologies which will have to withstand radiation levels of order $10^{16} 1 \mathrm{MeV}$ neutron equivalents $\mathrm{cm}^{-2}$.

Diamond is a promising material with remarkable mechanical and electronic properties to provide the basis for radiation resistant particle detectors for high energy particle physics (HEP). In the following the current state of diamond detectors in particle physics will be discussed and an outlook to future technologies to further enhance the radiation hardness will be given.

\section{Development of CVD Diamond Detectors}

The RD42 Collaboration has made significant progress over the last 20 years to improve the particle detection capabilities of synthetically produced diamond by closely working together with manufacturers. The signal yield for a minimum ionizing particle (MIP) has been increased to about 10000 electrons for a $500 \mu \mathrm{m}$ thick poly-crystalline diamond detector, and full charge collection is available for synthetic single crystal diamond. Currently, two manufacturers are offering commercially high quality poly-crystalline diamond material, namely ElementSix Ltd and II-VI Semiconductors. Detector grade single crystal diamond is available from ElementSix Ltd only.

\subsection{Diamond Detectors in HEP experiments}

Diamond detectors have been installed and successfully operated in Particle Physics experiments at the LHC and Tevatron experiments [1, 2, 3, 4, 5] in beam-monitoring applications.

At the CMS experiment diamonds have been used in the Beam Condition Monitor (BCM) [3, 4] . It consists of several detector systems to protect the silicon tracker of the CMS experiment from beam accidents, and provides feedback to the LHC machine operations. There are three diamond based sub-systems deployed: BCM1L and BCM2 use poly-crystalline CVD diamond and a beam-induced current measurement with integration times varying between $40 \mu \mathrm{s}$ to $83 \mathrm{~s}$. The BCM1L system consists of four diamonds at $4.5 \mathrm{~cm}$ distance from the beam pipe and at both sides $1.8 \mathrm{~m}$ from the interaction point. The BCM2 system consists of four diamonds at $5 \mathrm{~cm}$ distance from the beam pipe and eight diamonds at $28 \mathrm{~cm}$ distance from the beam pipe at both sides 14.4 $\mathrm{m}$ from the interaction point. Adverse beam conditions are detected by increased current values in the detectors. The BCM2 DC signal appeared to be sensitive, the cause of the DC current signal degradation with absorbed dose is under study. Additionally, a system based on single crystal diamond and fast read-out electronics is installed near BCM1L with four detector planes on each side of the CMS experiment. The BCM1F showed a linear behavior with luminosity.

The ATLAS experiment has had a similar system installed for run-1, and the performance was satisfactory to a degree that the BCM system became a detector component to determine the luminosity. 


\subsubsection{Upgrade plans}

The beam monitoring system of ATLAS was upgraded during the long shutdown between run-1 and run-2 with a Diamond Beam Monitor (DBM). The DBM consists of eight mini-trackers of three planes each using a pixel detector. Poly-crystalline diamond samples with a dimension of $18 \mathrm{~mm}$ by $21 \mathrm{~mm}$ are used. The signal from a minimum ionizing particle is measured to be above 9000 electrons. The sensors are bump-bonded to an FE-I4 [6] read-out chip. The system has been installed in fall 2013 in the ATLAS detector with the primary purpose to measure the bunch-bybunch luminosity with a precision of better than $1 \%$ per luminosity block, which has a length of about 1-2 minutes. The bump-bonding efficiency after thermal cycling for installed modules is above $>99.9 \%$.

\section{Radiation hardness}

The radiation tolerance of CVD diamond to different types of irradiation has been studied with protons at $25 \mathrm{MeV}, 70 \mathrm{MeV}, 300 \mathrm{MeV}, 800 \mathrm{MeV}$, and $24 \mathrm{GeV}$, and pions at $300 \mathrm{MeV}$. Before and after irradiation the signal response was tested in a test-beam with $120 \mathrm{GeV}$ protons. The signal response was tested with a strip-detector configuration at an electric field of $2 \mathrm{~V} \mu \mathrm{m}^{-1}$. The samples were exposed to ${ }^{90} \mathrm{Sr}$ to fill vacant traps and establish a well defined and stable condition prior to the measurement. In an application as e.g. pixel detector in the inner layer of an LHC experiment the ionising radiation field is sufficiently high to establish a stable condition within a few seconds. Reconstructed tracks were required to pass through the active area of the diamond strip detector and the charge signal was built from the two highest signals within ten strips around the track. The radiation damage is parameterized with a simple model using an effective damage constant $k_{\lambda}$ to describe the decrease of signal

$$
1 / \lambda=1 / \lambda_{0}+k_{\lambda} \Phi
$$

with $\lambda$ the "Schubweg" [7], $\lambda_{0}$ the initial value before irradiation, and $\Phi$ the integrated particle flux. For $24 \mathrm{GeV}$ protons a value of $k_{\lambda}=0.62 \pm 0.07 \cdot 10^{-18} \mu \mathrm{m}^{-1} \mathrm{~cm}^{-2}$ is found. Poly and single crystal diamond show a consistent damage constant. A summary of the irradiation results and deduced damage constants is given in Table 1.

Table 1: Summary of RD42 irradiation results for protons and pions at different energies expressed as relative damage constant $k_{\lambda}$ normalized to $24 \mathrm{GeV}$ protons.

\begin{tabular}{|c|c|c|}
\hline Particle & Energy & Relative $k_{\lambda}$ \\
\hline $\mathrm{p}$ & $24 \mathrm{GeV}$ & 1 \\
$\mathrm{p}$ & $800 \mathrm{MeV}$ & 1.7 \\
$\mathrm{p}$ & $70 \mathrm{MeV}$ & 2.7 \\
$\mathrm{p}$ & $25 \mathrm{MeV}$ & 4.2 \\
$\pi^{+}$ & $300 \mathrm{MeV}$ & 2.9 \\
\hline
\end{tabular}

The experimental results on $k_{\lambda}$ have been compared to a description using the Norget-RobinsonTorrens theorem to predict displacements per atom (DPA) with a displacement energy of 43.3 $\mathrm{eV}$ [8]. Reasonable agreement is observed for energies above $100 \mathrm{MeV}$ as shown in Figure 1. 


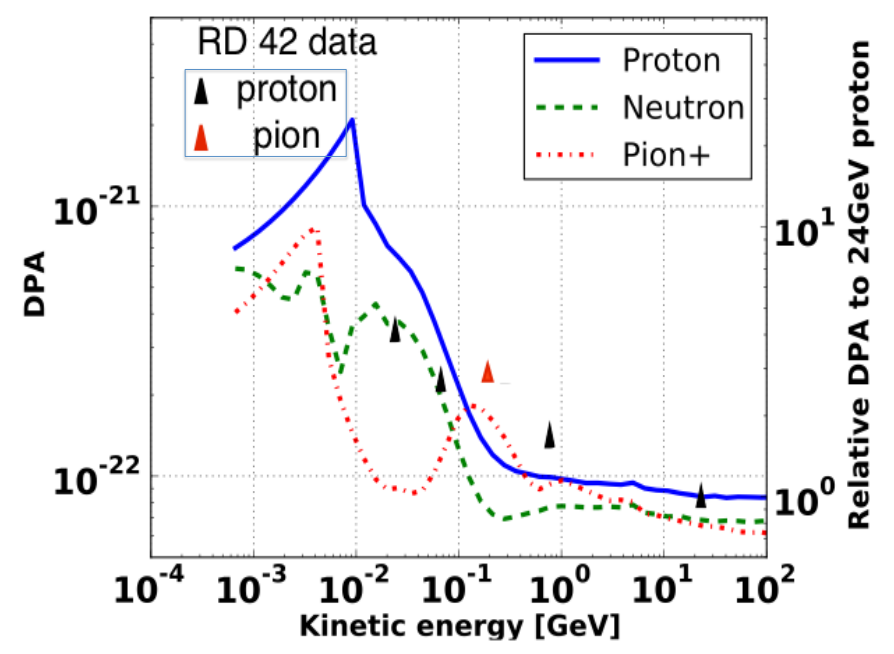

Figure 1: Comparison experimental data with theory prediction of the DPA for protons and pions at different energy in diamond.

\section{3D Diamond Detectors}

The radiation hardness of particle sensors can be improved by reducing the drift path needed to induce one unit charge as described by Ramo's theorem [9]. This can be achieved by e.g. reducing the thickness of the detector which has the disadvantage of a smaller signal due to the shorter ionization path. Another solution is to arrange the electrodes such that they penetrate the bulk of the active material, resembling the principle of a wire chamber but in a solid state detector. So called 3D detectors [10] keep the length of the ionization path equal to the thickness of the detector, but the drift path to induce one unit charge is given by the spacing of the electrodes in the bulk and can be much shorter. The 3D concept has been successfully demonstrated with Silicon detectors [11]. The same principle can be applied to diamond if a solution is found to fabricate electrodes in the bulk of the diamond substrate. Building on work that showed that a femto-second pulsed laser can be used to induce a localized phase-transition from diamond into an amorphous carbon phase [12], a laser process was employed to produce a first 3D diamond prototype.

This prototype device was constructed with a single crystal diamond substrate of $4.7 \mathrm{~mm}$ by $4.7 \mathrm{~mm}$ in size and a thickness of $500 \mu \mathrm{m}$, comprising three test structures, a simple planar strip structure with $50 \mu \mathrm{m}$ pitch, a 3D metallization pattern without bulk electrodes (3D phantom), and a full 3D prototype detector with 100 and $150 \mu \mathrm{m}$ pitch. The metallization pattern is shown in Figure 2. One can see the strip detector on the left, the 3D phantom structure in the middle, and the $3 \mathrm{D}$ detector on the right. The 3D detector has cubic cells with a base length equal to the pitch. The cells are ganged together to form columns. The pads at the bottom were connected to individual read-out channels of a VA2 low-noise CMOS amplifier array with 128 channels, analogue readout with DC coupling.

The structured metallization with $\mathrm{Cr}$-Au was applied using a photo-lithographic process. Conducting columns have been produced with a laser graphitisation process. The columns are penetrat- 
ing the full thickness of the diamond and have a diameter of about $6 \mu \mathrm{m}$ and a resistivity of about $1 \Omega \mathrm{cm}$ consistent with nano-crystalline graphite.

The strip and 3D phantom structures served as reference structures to verify that the signal is not entirely due to the surface metallization (3D phantom), and to compare the signal to the well understood planar strip geometry.

The prototype 3D detector was tested in a $120 \mathrm{GeV}$ proton beam at the CERN SPS. A silicon strip tracking telescope was used to define the location of particle tracks on the 3D sensor with a position resolution of the order of a few microns. The diamond strip detector was biased with $500 \mathrm{~V}$, the $3 \mathrm{D}$ structures with $25 \mathrm{~V}$. The strip detector showed a uniform response as expected. The 3D phantom showed a much lower response of about a third on average when compared to the strip detector. The 3D prototype structure shows a varying response due to missing electrodes (see Figure 3). The success rate of electrode production was about $90 \%$ leading to a high fraction of incomplete cells. To analyze the 3D detector performance a contiguous area of 18 cells was identified and used for the subsequent analysis. The response spectrum of this region showed a similar most-probable value as the strip detector as shown in Figure 3 thus proving that a $3 \mathrm{D}$ diamond detector can be built with the laser processing approach.

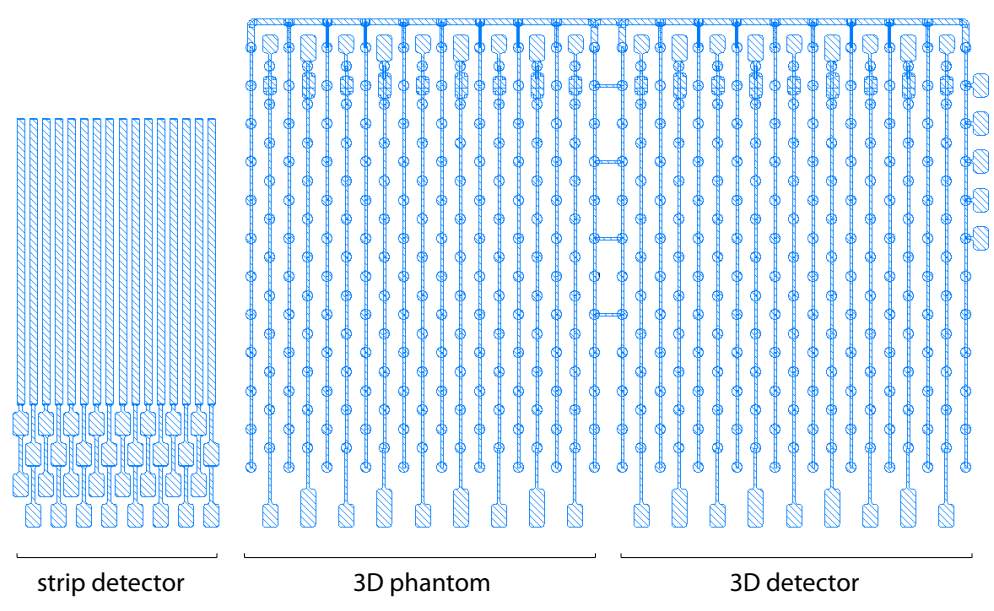

Figure 2: Metallisation pattern of the diamond prototype detector with the three test structures, namely strip detector, 3D phantom, and 3D detector. The 3D phantom does not contain 3D electrodes but just the surface metallisation, whereas the 3D detector contains 3D electrodes.

\section{Summary}

Diamond sensors are an established technology in the field of high energy particle physics. They have been successfully implemented in the beam monitoring systems of the ATLAS and CMS detectors. The next generation beam monitors based on diamond pixel sensors will measure the bunch-by-bunch luminosity to high precession and have been installed for the run-2 of the LHC. The radiation resistance has been tested with protons and pions over a wide energy range and 

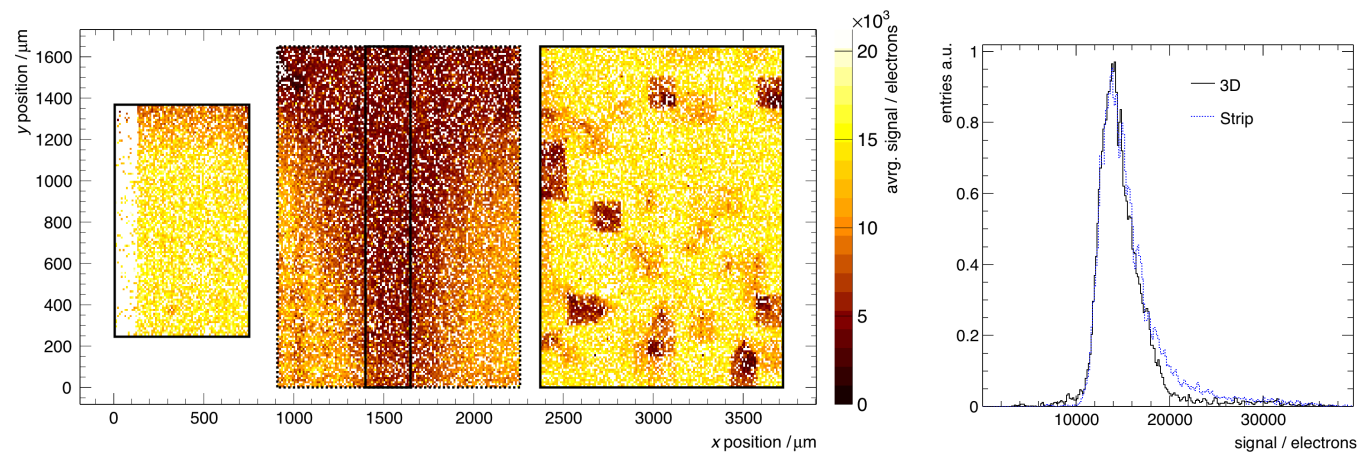

Figure 3: Average charge signal of the strip, 3D phantom and 3D prototype detector as a function of the position on the test device (left), and comparison of the signal spectrum of the strip and the 3D detector using the contiguous region (right).

the findings are parameterized with an effective damage model. To enhance further the radiation hardness 3D diamond sensors are being developed. A prototype 3D sensor based on a single crystal diamond has been successfully fabricated and tested.

\section{Acknowledgments}

$\mathrm{AO}$ would like to acknowledge support from the Royal Society.

\section{References}

[1] Cerv M. The ATLAS Diamond Beam Monitor. Geneva: CERN; 2013. ATL-INDET-PROC-2013-021.

[2] Alpigiani C, Gorisek A, Saxon J, Schorlemmer A, Valenitinetti S, Venturi N. ATLAS Tracking, Beam Protection and Forward Detector Systems. 2013 Apr;.

[3] Bell A, Castro E, Hall-Wilton R, Lange W, Lohmann W, Macpherson A, et al. Fast beam conditions monitor $\{\mathrm{BCM} 1 \mathrm{~F}\}$ for the $\{\mathrm{CMS}\}$ experiment. Nuclear Instruments and Methods in Physics Research Section A: Accelerators, Spectrometers, Detectors and Associated Equipment. 2010;614(3):433 - 438 .

[4] Mueller S, Boer W, Mueller T. The Beam Condition Monitor 2 and the Radiation Environment of the CMS Detector at the LHC. University of Karlsruhe. Karlsruhe, Germany; 2011. CERN-THESIS-2011-085 ; CMS-TS-2010-042; PhD Thesis, presented on 14 Jan 2011. Available from: http://cds.cern.ch/record/1319599.

[5] Sfyrla A, Eusebi R, Tesarek RT, Dong P, Schrupp C, et al. Beam Condition Monitoring with Diamonds at CDF. IEEE TransNuclSci. 2008;55:328-332.

[6] Garcia-Sciveres M, Arutinov D, Barbero M, Beccherle R, Dube S, Elledge D, et al. The FE-I4 pixel readout integrated circuit. Nuclear Instruments and Methods in Physics Research Section A: Accelerators, Spectrometers, Detectors and Associated Equipment. 2011;636(1, Supplement):S155 S159. 7th International Hiroshima Symposium on the Development and Application of Semiconductor Tracking Detectors.

[7] Hofstadter R. Crystal counters. Nucleonics. 1949;p. 2. 
[8] Guthoff M, de Boer W, Müller S. Simulation of beam induced lattice defects of diamond detectors using FLUKA. Nuclear Instruments and Methods in Physics Research Section A: Accelerators, Spectrometers, Detectors and Associated Equipment. 2014;A735:223-228.

[9] Ramo S. Currents Induced by Electron Motion. Proceedings of the IRE. 1939;27(9):584.

[10] Parker SI, Kenney CJ, Segal J. 3D - A proposed new architecture for solid-state radiation detectors. Nuclear Instruments and Methods in Physics Research Section A: Accelerators, Spectrometers, Detectors and Associated Equipment. 1997;395(3):328 - 343. Http://www.sciencedirect.com/science/article/pii/S0168900297006943.

[11] Da Via C, Deile M, Hasi J, Kenney C, Kok A, Parker S, et al. 3D Active Edge Silicon Detector Tests With $120 \mathrm{GeV}$ Muons. Nuclear Science, IEEE Transactions on. 2009 April;56(2):505-518.

[12] Kononenko TV, Meier M, Komlenok MS, Pimenov SM, Romano V, Pashinin VP, et al. Microstructuring of diamond bulk by IR femtosecond laser pulses. Applied Physics A. 2008;90(4):645-651. Http://dx.doi.org/10.1007/s00339-007-4350-9. 